\title{
Cascade Reservoir Optimal Operation Based On Chaotic Particle Swarm Optimization
}

\author{
Dunyu Zhong ${ }^{\mathrm{a}}$,Zengchuan Dong, ${ }^{\mathrm{a}}$, Yan Zhao ${ }^{\mathrm{b}}$, Wei Xu $^{\mathrm{a}}$, Xike Guan \\ ${ }^{a}$ College of Hydrology and Water Resources (Hohai University), Nanjing210098, China \\ ${ }^{\mathrm{b}}$ Yellow River Institute of Hydraulic Research, Zhengzhou 450003, China
}

Keywords: particle swarm optimization; chaotic search; cascade reservoir; optimal operation

\begin{abstract}
In order to solve the cascade reservoir system scheduling, this paper proposed a novel cascade reservoir optimal operation model based on chaotic particle swarm optimization (CPSO) algorithm. The traditional particle swarm algorithm is improved by using the nature of chaos sequence including the quasi-stochastic property and ergodicity, which can avoid particle swarm optimization (PSO) algorithm into local optimum and improve the speed and precision of the particle optimization process. The case study in the Upper Yellow River showed that the proposed model was capable of cascade reservoir optimal operation.
\end{abstract}

\section{Introduction}

Human societies and economic entities are seriously threatened by diverse factors, such as water and energy shortages, environmental degradation and global climate change. Therefore, there are urgent needs for sustainable water resources development and implementation of renewable energy strategies [1]. Hydropower has become a critical as it is renewable, clean, and cheap. The cascaded reservoirs are hydraulically coupled as the outflow of a reservoir constitutes part of the inflow into its immediate downstream reservoir. The optimal operation of the cascade reservoir is beneficial for the overall development of the river basin [2].

The multi-reservoir hydroelectric system scheduling is a class of large-scale, dynamic, nonlinear and non-convex constrained optimization problem in the economic operation of power systems [3]. Various optimization algorithms have been applied to solve the optimization problems related to the operation of cascade reservoir hydropower systems. The algorithms are usually classified into three general categories: traditional optimizers, modern metaheuristics, and hybrid approaches. Various optimization models based on linear programming (LP), no-linear programming (NLP), dynamic programming (DP), genetic algorithms (GA), artificial neural Network (ANN) and differential evolution (DE) have been developed for solving the cascade reservoir hydroelectric system scheduling problem in recent years [5, 6]. However, the existed drawbacks included premature phenomena and trapping in the local optimum lead to these methods no longer suitable for complex cascaded hydro system[5].
Particle swarm optimization (PSO) algorithm has been introduced by Kennedy and Eberhart and is a population based stochastic evolutionary method inspired by the emergent motion of a flock of birds searching for food [4] Due to the simple framework, easy implementation and quick convergence; PSO has attracted much attention in recent years. In the field of water resources, the PSO algorithm is also increasingly applied in parameter estimation for hydrological models, water supply planning and reservoir system operation [5]. However, it is easy to be trapped in local optimal point when dealing with some complicated engineering optimization problems. This paper proposes a novel chaotic particle swarm optimization (CPSO) algorithm to deal with Cascaded hydroelectric system dispatching problem in the Upper Yellow River. It is found that CPSO algorithm can provide better results to all models for decision-makers by choosing typical year data for optimization analysis.

\section{CPSO}

In a PSO system, the problem can be considered to be a bird (particles) in the solution space, particles fly around in the problem search space to look for the optimal solution. When a particle flight after a step at a time, particle adjusts its position according to its position according to its own "experience" as well as the experience of neighboring particles. The velocity and position of a particle updating equation is as follows.

$$
\begin{gathered}
v_{i j}(k+1)=w(k) v_{i j}(k)+c_{1} r_{1}\left(P_{i}(k)-X_{i j}(k)\right)+c_{2} r_{2}\left(G(k)-X_{i j}(k)\right) \\
X_{i j}(k+1)=X_{i j}(k)+v_{i j}(k+1)
\end{gathered}
$$

Where $w(k)$ is the inertia weight factor; $c_{1}$ and $c_{2}$ are the learning factors which influence particle movement within a generation. $k$ is the current iterative number; $X_{i j}(k)$ is the particle position in the $j$ dimension of the $i$ particle, $v_{i j}(k)$ is the velocity in the $j$ dimension of the $i$ particle; $v_{i j} \in\left[-v_{j \max }, v_{j \max }\right] ; r_{1}$ and $r_{2}$ are random values between 0 and $1 ; P_{i}(k)$ is the individual extreme value point; $G(k)$ is global extreme value point.

Chaotic optimization algorithm (COA), which utilizes the nature of chaos sequence including the quasi-stochastic property and ergodicity, is a popular optimization method[6] CPSO is introduction of chaos optimization ideas into particle swarm optimization algorithm; the main purpose is to find the 
dynamic perturbation of the particles by introducing the norepetitive ergodicity of the chaos into the solution space. By introducing processing, can make the particles in the solution space in the random and regular "flight", jump out from local space to find a better solution, improve the speed and precision of the particle optimization process. Steps of the CPSO are as follows: (a) Generates the initial particle swarm; (b) Update the particle status; (c) Each particle is calculated according to the fitness function, and the optimal one is selected as the optimal solution; (d) Chaotic perturbation of the optimal solution of population; (e) Stop CPSO if the terminating criteria is reached. Otherwise turn step (b).

\section{Reservoir Optimal Operation Model based on CPSO}

\subsection{Objective functions}

Power generation is a significant benefit derived from a cascade reservoirs system [8]. Cascade reservoirs operation optimization aims at maximizing the power generation by developing an optimal plan over the entire planning horizon, while satisfying all kinds of physical and operational constraints. However, in the pursuit of the largest power generation indicators will ignore the role of reservoirs in the dry season, resulting in significant changes in the output of the turbine during the year. Therefore, this study chooses to maximize the guaranteed output for the objective during the calculation period. For the convenience of calculation, the maximum guaranteed output converted to the actual output as close as possible to equal the installed capacity. The objective function can be formulation as follows.

$$
\Delta N=\min \sum_{t}\left(\frac{\sum_{i=0}^{n} N_{i t}-N_{y}}{N_{y}}\right)^{2}
$$

Where $N_{i t}$ denotes output of the $i$ th hydropower station in the $t$ th stage, $N_{y}$ denotes The upstream cascade reservoirs slot installed capacity. Reservoir indexes from upstream to downstream are $1,2, \ldots, \mathrm{n}$ in this paper.

\subsection{Constraint conditions}

(1)Water balance equation:

$$
V_{i t}-V_{i, t-1}=\left(I_{i t}-Q_{i t}-E_{i t}\right) \Delta t
$$

where $V_{i t}$ and $V_{i, t-1}$ are the initial storages of the $i$ th reservoir at times $t$ and $t$ - 1 , respectively; $I_{i t}$ is the inflow of the $i$ th reservoir at time $t ; Q_{i t}$ is the outflow of the $i$ th reservoir at time $t ; E_{i t}$ is the Loss of flow of the $i$ th reservoir at time $t$; and $\Delta t$ is the duration.

(2)Outflow

$$
Q_{i t, \min } \leq Q_{i t} \leq Q_{i t, \max }
$$

Where $Q_{i t, \min }$ and $Q_{i t, \max }$ are the minimum and maximum allowable outflow of the $i$ th reservoir at time $t$, respectively. (3)Water level

$$
Z_{i t, \min } \leq Z_{i t} \leq Z_{i t, \max }
$$

Where $Z_{i t \text {,min }}$ and $Z_{i t \text {,max }}$ are the dead level and the maximum water level of the $i$ th reservoir at time $t$, respectively.

(4)Hydropower generation

$$
N_{i t, \min } \leq N_{i t} \leq N_{i t, \max }
$$

Where $N_{i t}, N_{i t, \min }$ and $N_{i t, \max }$ are the output, minimum output and maximum output of hydropower produced from the $i$ reservoir at time $t$, respectively.

(5)Ecological flow

In order to avoid zero-flow problems, a certain amount of flow in each river section must be preserved to maintain the ecological balance of the River.

$$
R_{i t} \geq R_{i t, \min }
$$

Where $R_{i t \text {, min }}$ is the minimum flow of the $i$ th section at time $t$.

(6) Ice control

$$
R_{t, \min }^{*} \leq R_{t} \leq R_{t, \max }^{*}
$$

Where $R_{t, \text { min }}^{*}$ and $R_{t, \text { max }}^{*}$ are the minimum and maximum ice control outflow at mouth $t$, respectively.

(7) Variable nonnegative constraints

\subsection{Initial solution selection}

When using the CPSO optimization algorithm, each particle contains the information of multiple reservoirs at the same time. Establish the particle group consists of $\boldsymbol{m}$ particles, $i$ is the particle number, $K$ is the particle group co-evolution $\mathrm{K}$ generation. The upstream water level of each reservoir was taken as the decision variable, and the position vector of each particle is represented by this water level sequence, where $Z_{i, t}^{d}(k)$, the element of $X_{i}(k)$, is the water level for the $k$ th population $(1 \leq k \leq K)$, for the $\boldsymbol{i}$ th particle $(1 \leq i \leq m)$ at the end of $t(1 \leq t \leq 2 T)$ time period of reservoir $d$, where $d$ is the reservoir serial number and $T$ represents the calculation period of a reservoir. In this paper, the calculation of the step length of the month, the calculation cycle of the year. Consequently, the particle expression takes the following form:

$$
X_{i}(k)=\left(Z_{i, 1}^{1}(k), \ldots, Z_{i, t}^{1}(k), \ldots, Z_{i, T}^{1}(k), Z_{i, 1}^{2}(k), \ldots, Z_{i, t}^{2}(k), \ldots, Z_{i, T}^{2}(k)\right)
$$

The speed vector $v_{i}(k)$ indicates the fluctuation rate of the water level at the end of each time period of the reservoir, where $v_{i, t}^{d}(k)$, the element of $v_{i}(k)$, is the fluctuation rate for the $k$ th population $(1 \leq k \leq K)$, for the $i$ th particle $(1 \leq i \leq m)$ at the end of $t(1 \leq t \leq 2 T)$ time period of reservoir $d$. The expression takes the following form: 


$$
v_{i}(k)=\left(v_{i, 1}^{1}(k), \ldots, v_{i, t}^{1}(k), \ldots, v_{i, T}^{1}(k), v_{i, 1}^{2}(k), \ldots, v_{i, t}^{2}(k), \ldots, v_{i, T}^{2}(k)\right)
$$

The initial solution is generated to meet certain constraints to ensure the feasibility of the trajectory at each time period. The initial feasible solution of each reservoir can be determined by changing the flow constraints to water level constraints by the range of reservoir discharge flow. The specific methods are given as follows:

(1) Determination the water level range in the time period At the initial moment, the reservoir starts from the original regulating water level (i.e., $V_{\mathrm{O}}{ }^{d}$ is known), depending on the requirements of the minimum discharge limit $Q_{\min }{ }^{d}(t)$ and the maximum discharge limit $Q_{\max }{ }^{d}(t)$ when $t=1$. Then the minimum $V_{t, \text { min }}^{d}$ and the maximum reservoir storage $V_{t, \text { max }}^{d}$ can be calculated according to the water balance equation, when the inflow $I$ is known. And then the corresponding minimum $Z_{t, \min }{ }^{d}$ and maximum water level $Z_{t, \max }{ }^{d}$ are obtained by finding the water level storage capacity curve.

(2) Obtaining the eigenvalues of the initialized particles by using the chaotic series

Let $\mathrm{k}=1$, generate two sets of $\mathrm{N}$-dimensional $(N=2 T)$ vectors between $[0,1]$ using the random function, corresponding to the position and velocity of the first particle, respectively:

$$
\begin{aligned}
& \beta_{1}=\left(\beta_{11}^{1}(1), \beta_{12}^{1}(1), \ldots, \beta_{1 T}^{1}(1), \beta_{11}^{2}(1), \beta_{12}^{2}(1), \ldots, \beta_{1 T}^{2}(1)\right) \\
& \mu_{1}=\left(\mu_{11}^{1}(1), \mu_{12}^{1}(1), \ldots, \mu_{1 T}^{1}(1), \mu_{11}^{2}(1), \mu_{12}^{2}(1), \ldots, \mu_{1 T}^{2}(1)\right)
\end{aligned}
$$

Where each component $\beta_{1 t}{ }^{d}(1), \mu_{1 t}{ }^{d}(1)$ indicates the random value corresponding to the water level and the water level fluctuation velocity at the end of $t(1 \leq t \leq 2 T)$ time period of reservoir $d$ in the first particle of the initial population (i.e., the first generation population), respectively. (3) carrying $\beta_{i}, \mu_{i}$ to an optional range $\left[Z_{t, \min }^{d}, Z_{t, \max }^{d}\right]$

The various components of $\beta_{i}, \mu_{i}$ are carried to the range of the reservoir water level variable and its fluctuation speed:

$$
\begin{gathered}
Z_{i t}{ }^{d}(k)=Z_{t, \text { min }}{ }^{d}+\left(Z_{t, \text { max }}{ }^{d}-Z_{t, \text { min }}{ }^{d}\right) \beta_{i t}{ }^{d}(k) \\
v_{i t}{ }^{d}(k)=v_{t, \min }{ }^{d}+\left(v_{t, \text { max }}{ }^{d}-v_{t, \text { min }}{ }^{d}\right) \mu_{i t}{ }^{d}(k)
\end{gathered}
$$

Where $Z_{t, \min }{ }^{d}$ and $Z_{t, \max }{ }^{d}$ represent the minimum and the maximum water level of the end of $t(1 \leq t \leq 2 T)$ time period of reservoir $d$, respectively. That is, the water level ranges of the reservoir $\left[Z_{t+1, \min }{ }^{d}, Z_{t+1, \max }{ }^{d}\right]$ determined by the previous step. While $v_{t, \min }{ }^{d}, v_{t, \max }{ }^{d}$ respectively refer to the minimum and the maximum water level fluctuation velocity of the end of $t(1 \leq t \leq 2 T)$ time period of reservoir $d$, where $v_{t, \min }{ }^{d}=-v_{t, \max }{ }^{d}, v_{t, \max }{ }^{d}$ is the 0.1 of the difference between the maximum and the minimum values, as given by:

$$
v_{t, \max }^{d}=0.1 \times\left(z_{t, \max }{ }^{d}-z_{t, \min }{ }^{d}\right)
$$

When $t=1, d=1$, the water level $Z_{11}{ }^{1}(1)$ generated by the first particle carrier can be used as the end of first time period of the water level.

(4) Formation of water level at each end of the time period The water level at $\mathrm{t}=1$ is taken as the initial water level for the next time period, and then repeat the above three steps, calculate it until the end of the time interval time period by time period. And a reservoir initial operation scheduling trajectory can be obtained by connecting the random water level generated according to a certain regular at each time interval.

\section{Its Application}

This study selected seven reservoirs (longyangxia, laxiwa, lijiaxia, gongboxia, liujiaxia, yanguoxia, qingtongxia) as the research object in the Upper Yellow River basin (see fig. 1). LYX reservoir is a multi-year regulating reservoir; LJX Reservoir is an annual regulating reservoir; therefore, these two reservoirs need to consider their regulatory role in the process of reservoir optimization. While others are Runoff regulation ability, only consider the usage of water head power rather than the function of regulation.

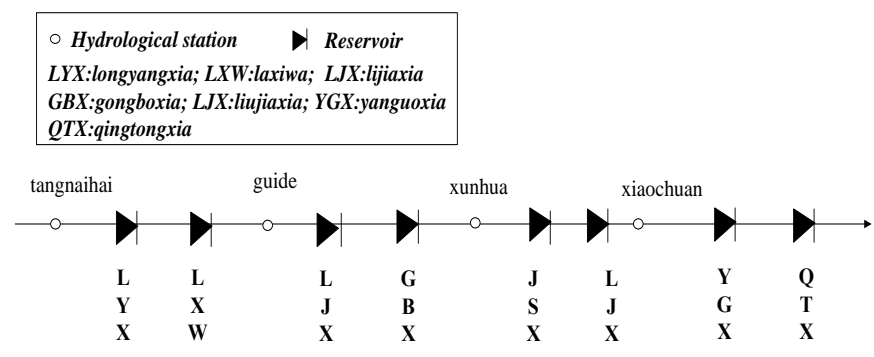

fig. 1: Reservoir generalization of Upper Yellow River

The model scheduling period is one year and calculation period is a month; the beginning water level and the ending water level of the reservoir are the same as the actual year (Table I ). Only optimize the middle month. The LYX beginning water level is $2580.06 \mathrm{~m}$ and the ending water level is $2572.11 \mathrm{~m}$; the $L J X$ beginning water level is $1721.55 \mathrm{~m}$ and the ending water level is $1721.27 \mathrm{~m}$.

CPSO is used to solve the optimal operation of cascade reservoir. According to the actual water level of the reservoir set the model parameters. The parameters of CPSO are set as follows. The population size is set to $\mathrm{m}=100$, the inertia weight factor $w(k)$ linearly decreases from $w_{\max }=0.9$ to $w_{\text {min }}=0.4$ using $c_{1}=c_{2}=2$, the maximum generation iteration is set to Maxgen $=1000$, the maximum iterative number of chaotic search is $K_{\max }=100$. The algorithm is realized by using VB6.0. Select July 2010 - June 2011 data to optimize the scheduling calculation and analysis of the results to verify the model optimization effect (Table II ). From Table 2, we could find that, the optimal power generation of reservoirs is higher than the actual power generation, and the total power of the cascade is increased by $2.9 \%$. 


\begin{tabular}{|l|c|c|c|c|c|c|c|c|}
\hline Item & unit & LYX & LXW & LJX & GBX & LJX & YGX & QTX \\
\hline Dead water level & $\mathrm{m}$ & 2530 & 2440 & 2178 & 2002 & 1717 & 1618 & 1151 \\
\hline Normal water level & $\mathrm{m}$ & 2600 & 2452 & 2180 & 2005 & 1735 & 1619 & 1156 \\
\hline Total storage capacity & $10^{8} \mathrm{~m}^{3}$ & 247 & 10.6 & 11.7 & 5.5 & 57 & 2.2 & 5.7 \\
\hline Regulation ability & - & Multi-year & Runoff & Runoff & Runoff & Annual & Runoff & Runoff \\
\hline Installed capacity & $10^{4} \mathrm{~kW}$ & 128 & 420 & 200 & 150 & 135 & 47 & 30.2 \\
\hline Efficiency coefficient & - & 8.3 & 8.5 & 8.5 & 8.5 & 8.3 & 7.9 & 8.4 \\
\hline Guaranteed output & $10^{4} \mathrm{~kW}$ & 58.98 & 99 & 58.98 & 49.2 & 48.99 & 20.5 & 9.09 \\
\hline Maximum outflow & $\mathrm{m}^{3} / \mathrm{s}$ & 1200 & 2280 & 1900 & 1675 & 1400 & 1400 & 2106 \\
\hline
\end{tabular}

Table I : Basic Information of reservoir in the Upper Yellow River

\begin{tabular}{|l|c|c|c|c|c|c|c|c|}
\hline Item & LYX & LXW & LJX & GBX & LJX & YGX & QTX & Total \\
\hline Calculate power generation & 63.13 & 107.66 & 64.81 & 53.23 & 60.79 & 20.93 & 13.60 & 384.15 \\
\hline Actual power generation & 61.66 & 106.10 & 64.05 & 52.49 & 57.33 & 20.88 & 10.94 & 373.45 \\
\hline
\end{tabular}

Table II : Scheduling results based on CPSO $\left(10^{8} \mathrm{kWh}\right)$

\section{Conclusions}

This paper puts forward the CPSO is used to solve cascade reservoir of scheduling optimization, and based on CPSO a cascade reservoir optimal model was set up and applied in the Upper Yellow River. The algorithm has the following advantages. Fast calculation, high search efficiency; Good convergent performance, easy to jump out of local optimal solution; principle is simple, easy to programming. The results show that the model is reasonable and the algorithm is feasible, and it can be used to describe the cascade reservoir scheduling. This study provides important recommendations on sustainable management and unified allocation of water resources over the Yellow River basin and a sound scientific basis for optimizing the operation of cascade reservoirs. Combined with the CPSO to solve the multi-objective optimization scheduling problem is the next step.

\section{Acknowledgements}

This work was supported by the project(2016-005-HHS-KJ-X) supported by the Yellow River Upper Reach Hydroelectric Exploitation Co Ltd, China, the project(2016YFC0401306) supported by the National Key Research and Development Program, China.

\section{References}

[1] Lund H, Afgan H, Bogdan Z, et al. Renewable energy strategies for sustainable development, Energy, 32, pp. 912919, (2007).

[2] Zhang X, Yu X, Qin H. Optimal operation of multireservoir hydropower systems using enhanced comprehensive learning particle swarm optimization. Journal of Hydroenvironment Research, 10, pp. 50-63, (2016).
[3] He Y, Yang S, Xu Q. Short-term cascaded hydroelectric system scheduling based on chaotic particle swarm optimization using improved logistic map. Communications in Nonlinear Science \& Numerical Simulation, 18, pp. 17461756, (2013).

[4] Kennedy J, Eberhart R. Particle swarm optimization// IEEE International Conference on Neural Networks, Proceedings. IEEE Xplore, pp. 1942-1948, (1995).

[5] Bonin O, Rousseaux F. Multireservoir systems optimization using genetic algorithms : Case study. Journal of Computing in Civil Engineering, 14, pp. 255-263, (2000).

[6] Chandramouli V, Raman H. Multireservoir Modeling with Dynamic Programming and Neural Networks. Journal of Water Resources Planning \& Management, 127, pp. 89-98, (2001).

[7] Zhang J, Zhen W U, Cheng C T, et al. Improved particle swarm optimization algorithm for multi-reservoir system operation. Water Science and Engineering, 4, pp. 61-73, (2011).

[8] Zhang Y, Jiang Z, Ji C, et al. Contrastive analysis of three parallel modes in multi-dimensional dynamic programming and its application in cascade reservoirs operation. Journal of Hydrology, 529, pp. 22-34, (2015). 